\title{
Challenges in the nursing care of intracranial carbapenem- resistant Escherichia coli infection after severe traumatic brain injury: a case report
}

\author{
Ping Wang ${ }^{1}$, Bingbing Gao ${ }^{1}$, Ming Wang ${ }^{1}$, Qingyu Sheng ${ }^{1}$, Mingmin Tu ${ }^{2}$ \\ ${ }^{1}$ Department of Neurosurgery, The First Affiliated Hospital, College of Medicine, Zhejiang University, Hangzhou, China; ${ }^{2}$ Shulan (Hangzhou) \\ Hospital Affiliated to Zhejiang Shuren University Shulan International Medical College, Hangzhou, China \\ Correspondence to: Mingmin Tu. Shulan (Hangzhou) Hospital Affiliated to Zhejiang Shuren University Shulan International Medical College, \\ Hangzhou, China. Email: 1200038@zju.edu.cn.
}

\begin{abstract}
This article described a case of intracranial carbapenem-resistant Escherichia coli (CRE) infection after severe traumatic brain injury (TBI) and summarized the nursing experience. Since the intracranial infection was confirmed in the early stage and added secondary intracranial hypertension will directly affect the prognosis, the responsible nurse is required to increase the frequency of ward patrol, carefully observe the patient's consciousness and pupil changes, monitor vital signs and improve relevant records, communicate with the competent doctor in time, use drugs, or physical cooling to control high fever as soon as possible. Intensive observation and nursing care included: (I) position management; (II) observation on the state of illness during injection; (III) observation on the state of illness during tube clamping period; (IV) nursing after recovery of drainage; and (V) drug treatment after removing the drainage tube of the lumbar cistern. Artificial airway management included (I) position management, (II) periodically evaluate the artificial airway, (III) airway humidification and warming, (IV) management over airbags, (V) aspirate sputum as needed and (VI) oral care. With appropriate antibiotic drugs, reasonable arrangements to observe the curative effect, and cooperation with doctors, targeted artificial airway management to reduce the risk of airway infections, we treated this patient effectively and successfully. The intracranial infection was effectively controlled after 15-day treatment and nursing in our department, and the patient received an excellent prognosis finally.
\end{abstract}

Keywords: Traumatic brain injury (TBI); carbapenem-resistant Escherichia coli (CRE); intracranial infection; nursing

Submitted May 18, 2020. Accepted for publication Jul 10, 2020.

doi: $10.21037 /$ apm-20-1264

View this article at: http://dx.doi.org/10.21037/apm-20-1264

\section{Introduction}

Intracranial infection is a common complication after traumatic brain injury (TBI) usually aggravates the patient's condition with the primary disease and increases mortality. Most patients died of intracranial hypertension or multiple organ failure (1). Treatment should focus on preventing secondary injury by avoiding hypotension and hypoxia and maintaining appropriate cerebral perfusion pressure. In the past 20 years, carbapenem-resistant Escherichia coli (CRE) has become a global public health problem with widespread rapid increase. Resulting in high morbidity, disability, and mortality, CRE brings considerable challenges to clinical treatment and severe burdens to society $(2,3)$. It is much easier for the elderly, immunocompromised and critically ill patients to infect CRE. The mortality rate is between $40 \%$ and $50 \%$ for severe patients with CRE bloodstream infections (4). In order to effectively decrease case-fatality rates among patients with the infections owing to CRE infection, combination antibiotic schemes, including colistin 
(or amikacin) and/or tigecycline in combination with an anti-pseudomonal carbapenem agent, remain the mainstay for treating clinical CRE infections. In September 2019, our department of neurosurgery treated a patient infected by intracranial CRE after severe TBI successfully. At present, few cases of intracranial infection caused by CRE have been reported. The successful treatment need intensive collaboration of doctors and nurses.

We present the following case in accordance with the CARE reporting checklist (available at http://dx.doi. org/10.21037/apm-20-1264).

\section{Case presentation}

A 67-year-old male patient was admitted to our neurosurgery department for intracranial carbapenemresistant Escherichia coli infection confirmed by culture after craniotomy on September 13, 2019. Fourteen days ago, the patient underwent craniotomy and bone flap decompression due to a right intracerebral hemorrhage after a traffic accident. Physical examination revealed that the Glasgow Coma Score (GCS) of the patient was E4 + T + M5. The wound did not heal well, with pus flowing out, which was cultured. Blood workup showed that the white blood count was $14.5 \times 10^{9}$ cell/L, and a highly sensitive C-reactive protein level was $118.29 \mathrm{mg} / \mathrm{L}$. Lumbar cistern drainage was performed at once at admission. The color of cerebrospinal fluid (CSF) was yellow and turbid, and CSF examination revealed. The laboratory results showed there were 5,700/UL nucleated cells, pan type test $(+++)$. The patient was started on polymyxin $\mathrm{B}$, tigecycline, meropenem triple antibiotics, with intrathecal injection of polymyxin B. After 5 days of continuous treatment, intravenous antibiotics were substituted with amikacin, tigecycline and meropenem according to the results of CSF culture On September 28, the intracranial infection was effectively controlled. The CSF workup showed a nucleated cell of 60/UL, pan test (-), no pathogenic bacteria in CSF culture. The patient's consciousness improved, with GCS of $\mathrm{E} 4+\mathrm{T}+\mathrm{M} 6$. Then, the patient was off respiratory support 5 days after admission. Under the gradual implementation of rehabilitation exercise, the muscle strength of both legs of the patient gradually improved. The muscle strength of the left leg was level III, and the right leg was level II. The patient was transferred to a rehabilitation hospital on October 7.

Written informed consent was obtained from the patient. All procedures performed in studies involving human participants were in accordance with the ethical standards of the institutional and/or national research committee(s) and with the Helsinki Declaration (as revised in 2013).

\section{Nursing}

\section{Prevention of intracranial bypertension}

The intracranial infection was confirmed in the early stage; added secondary intracranial hypertension will directly affect the prognosis. Therefore, the responsible nurse is required to increase the frequency of ward patrol, carefully observe the patient's consciousness and pupil changes, monitor vital signs and improve relevant records, communicate with the competent doctor in time, use drugs, or physical cooling to control high fever as soon as possible. In terms of drug lowering intracranial pressure, according to the doctor's order frequency, we correctly arrange the time and speed of mannitol infusion, observe the changes of urine volume, blood pressure, and electrolyte levels, to promptly handle and maintain the internal environment, to ensure adequate perfusion of brain tissue, and prevent the complication of intracranial hypertension or cerebral hernia.

\section{Rational use of antibiotics}

\section{Strict management of antibiotics treatment}

Antimicrobial treatment of extensively resistant gramnegative bacterial infections includes polymyxin, aminoglycosides, tigecycline, and fosfomycin. The efficacy of carbapenem combined with polymyxin in the treatment of CRE infection is better than a single drug or other combined programs (5). Some studies have shown that tigecycline has a high sensitivity of $97.4 \%$ in the treatment of CRE infection in adults (6-8). Tigecycline combined with polymyxin can effectively reduce the 30 days mortality of CRE infection patients. CSF culture-confirmed Escherichia coli infection in our case. After admission, he was started on tigecycline $50 \mathrm{mg}$, polymyxin B 750,000 UI, meropenem $2.0 \mathrm{~g}$, and polymyxin B 750,000 UI. According to the drug sensitivity results, the treatment plan was substituted with tigecycline $100 \mathrm{mg}$ intravenous drip for $12 \mathrm{~h}$, amikacin $600 \mathrm{mg}$ intravenous drip QD, and amikacin $30 \mathrm{mg}$ intrathecal injection. Amikacin $400 \mathrm{mg}$ IV drip/bid was used for 3 days, and the intrathecal dose of amikacin was adjusted to $50 \mathrm{mg}$ per day for 7 days. A responsible nurse needs to have a complete understanding of the treatment purpose 
and medication mode. During treatment, antibiotics are adjusted frequently and dynamically, so the nurse should strictly follow the drugs, guarantee the concentration of the drugs, reasonably distribute the drugs to improve the antibacterial effect. Simultaneously, we should pay attention to the CSF drainage, monitor the change of body temperature, conduct physical cooling and drug cooling in time according to the doctor's order, and observe the results of CSF and sputum culture. The body temperature returned to normal 5 days after admission, and the appearance of CSF was clear, meaning it had no abnormalities 10 days later in our case. No pathogenic bacteria were detected in the succeeding four CSF cultures, which proved that the antibacterial treatment was effective

\section{Nursing of intrathecal injection}

(I) Position management (9) To support a specific effective concentration in CSF circulation, the intrathecal injection was performed. The patient was placed in the supine position, a soft pillow placed on the back of their waist, and kept in this position for 1.5 hours during the injection.

(II) Observation on the state of illness during injection With the cooperation of medical care and strict aseptic operation, a $5 \mathrm{~mL}$ solution was injected by the pump and maintained for 30min. During the injection, ECG monitoring was given, vital signs were monitored, consciousness, pupil, and systemic reaction were observed.

(III) Observation on the state of illness during tube clamping period After injection, the patient took a flat position. The soft pillow on the back was removed. The pupil, consciousness, and vital signs were observed every 15 minutes during the clamping period.

(IV) Nursing after recovery of drainage The patient's head elevation of the bed was set to a position of $15^{\circ}$ and $45^{\circ}$ lying on the side. Nurses assisted the doctor to adjust the position of the drainage tube of the lumbar cistern, and properly fixed it, check the drainage tube every 4 hours to ensure the sealing and patency of the drainage, and observe the color, character, and quantity of the drainage fluid. During drainage, drainage flow was controlled to (200-300) $\mathrm{mL} / \mathrm{d}$, according to the doctor's instructions to avoid excessive drainage and secondary intracranial hypotension.

(V) Drug treatment after removing the drainage tube of the lumbar cistern. After the drainage tube of the lumbar cistern was removed, a lumbar puncture was conducted on 2/10, 4/10 and 7/10 days, respectively. CSF was kept for examination, and amikacin was injected intrathecally at a dose of $50 \mathrm{mg} / \mathrm{time}$.

\section{Observation of the side effects of drugs}

For the nephrotoxicity and neurotoxicity of polymyxin, the patient presented obvious neurotoxic reactions, including the muscle strength of both lower limbs decreasing, the muscle strength of grade I in the left lower limb, grade 0 in the right lower limb, after intrathecal injection. Therefore, polymyxin B was substituted with amikacin. Additionally, the patient received passive movement and underwent exercise treatment 2 times/day on the bed. The muscle strength of both legs gradually improved, with grade III in the left leg and grade II in the right leg, without muscle atrophy during admission.

\section{Artificial airway management}

\section{Position management}

Some studies have shown that (9) bed head elevation of $15-30$, sputum suction $45^{\circ}$ lateral position is not only beneficial for clearing airway secretion but also beneficial for improving brain oxygen metabolism and cerebrovascular permeability, relieving brain edema and reducing intracranial pressure for patients with a tracheotomy. To avoid excessive drainage of CSF during lumbar cistern drainage, the patient took a position of $15^{\circ}$ elevation of the bed head and $45^{\circ}$ lying on his side. The raised position of the bed head also helps prevent aspiration during enteral nutrition.

\section{Periodically evaluate the artificial airway}

Nurses should observe whether the artificial airway is unobstructed, the catheter is fixed properly, the pressure of the airbag is suitable, and the patient has asthma, cyanosis of the mouth and lip. Do not over pull the tracheotomy tube in the process of turning over and percussing the back, oral care, and sputum suction to prevent the displacement. The airbag pressure gauge is used to measure the airbag pressure to ensure safety and accuracy. The airbag pressure is monitored every 4 hours and supported at $25-30 \mathrm{cmH}_{2} \mathrm{O}$ (10), to seal the air passage and prevent aspiration effectively.

\section{Airway humidification and warming}

The artificial airway destroys the normal physiological respiratory tract, which affects the integrity of the airway mucosa (11), increasing the risk of respiratory inflammation $(12,13)$. Effective airway humidification can 
prevent respiratory infection. After admission, the patient underwent a tracheotomy and daily respiratory exercise. After 5 days, the patient was successfully weaned. No matter in using a ventilator and off-line, we always use HME to humidify and warm to prevent the formation of a sputum scab and avoid complications, including high airway response. This measure significantly reduces the amount of sputum secretion and the number of sputum suction. During the humidification heat exchanger period, the nurse in charge visited the ward every two hours to observe the humidification temperature and water level scale. These procedures were done to prevent the humidification effect from being affected by the drying of distilled water.

\section{Management over airbags}

The residue above the airbag is an important source of ventilator-associated pneumonia. The patient uses the washable air cutting sleeve and uses the low negative pressure suction under the glottis to remove the retention on the airbag every $\mathrm{Q}_{2}$ hours. We should note the 24 suction volume on the nursing record sheet and implement the shift handover system. When it is found the volume is less on a certain day, find out the reasons, and deal with them in time. For example, we check the position of the gas cutting sleeve and ask the performer whether to conduct suction as required.

\section{Aspirate sputum as needed}

An inspection tour is performed every hour. When obvious phlegm sound is heard in the respiratory tract or the blood oxygen saturation suddenly decreases, the patient should receive sputum aspiration at once. When there is no special situation, sputum suction should be conducted as needed. Before sputum suction, percussion and tremor should be conducted in the lungs. Chest physical treatment should be conducted to ensure the drainage and suction effect of sputum. Sputum aspiration should follow the aseptic operation system.

\section{Oral care}

High quality of oral care can prevent VAP (14). For this patient, we check the oral mucosa every shift. When the pressure of the airbag is checked to be safe with the salivary absorbing the toothbrush, rinse the mouth with chlorhexidine tinidazole gargle every 6 hours. During the process of rinsing, the movement is gentle to prevent mucosal bleeding. The contents of each oral care record, including patients' gums, teeth, oral mucosa, are reflected in the nursing record sheet to ensure that each shift can understand the current oral situation of the patient.

\section{Summary}

CRE infection brings a series of social problems, including clinical treatment failure, prolonged hospitalization, high medical cost, and increasing social burden (15). At present, few cases of intracranial infection caused by CRE have been reported. The successful treatment of this case includes timely and correct diagnosis and treatment, targeted nursing practice, prompt lumbar cistern CSF drainage to eliminate intracranial inflammatory substances, and accelerate CSF circulation (16). The puncturing process and clinical nursing process follow the sterile principle, avoiding secondary infection. The patient was satisfied with our work. In conclusion, full awareness of the clinical problems, cooperation in the medical team, prompt and effective treatment are key to a satisfactory prognosis, even in the disease of high mortality.

\section{Acknowledgments}

Funding: None.

\section{Footnote}

Reporting Checklist: The authors have completed the CARE reporting checklist. Available at http://dx.doi.org/10.21037/ apm-20-1264

Conflicts of Interest: All authors have completed the ICMJE uniform disclosure form (available at http://dx.doi. org/10.21037/apm-20-1264). The authors have no conflicts of interest to declare.

Ethical Statement: The authors are accountable for all aspects of the work in ensuring that questions related to the accuracy or integrity of any part of the work are appropriately investigated and resolved. Written informed consent was obtained from the patient. All procedures performed in studies involving human participants were in accordance with the ethical standards of the institutional and/or national research committee(s) and with the Helsinki Declaration (as revised in 2013).

Open Access Statement: This is an Open Access article distributed in accordance with the Creative Commons 
Attribution-NonCommercial-NoDerivs 4.0 International License (CC BY-NC-ND 4.0), which permits the noncommercial replication and distribution of the article with the strict proviso that no changes or edits are made and the original work is properly cited (including links to both the formal publication through the relevant DOI and the license). See: https://creativecommons.org/licenses/by-nc-nd/4.0/.

\section{References}

1. Collins-Praino L, Corrigan F. Cerebrovascular contribution to dementia development after traumatic brain injury: promises and problems. Ann Transl Med 2018;6:S58.

2. Ray MJ, Lin MY, Weinstein RA, et al. Spread of Carbapenem-Resistant Enterobacteriaceae Among Illinois Healthcare Facilities: The Role of Patient Sharing. Clin Infect Dis 2016;63:889-93.

3. Tijet N, Muller MP, Matukas LM, et al. Lateral dissemination and inter-patient transmission of blaKPC-3: role of a conjugative plasmid in spreading carbapenem resistance. J Antimicrob Chemother 2016;71:344-7.

4. De Rosa FG, Corcione S, Cavallo R, et al. Critical issues for Klebsiella pneumoniae KPC-carbapenemase producing K. pneumoniae infections: a critical agenda. Future Microbiol 2015;10:283-94.

5. Sader HS, Castanheira M, Farrell DJ, et al. Tigecycline antimicrobial activity tested against clinical bacteria from Latin American medical centres: results from SENTRY Antimicrobial Surveillance Program (2011-2014). Int J Antimicrob Agents 2016;48:144-50.

6. Martin A, Fahrbach K, Zhao Q, et al. Association Between Carbapenem Resistance and Mortality Among Adult, Hospitalized Patients With Serious Infections Due to Enterobacteriaceae: Results of a Systematic Literature Review and Meta-analysis. Open Forum Infect Dis 2018;5:ofy150.

7. Yang H, Chen G, Hu L, et al. Enhanced efficacy of

Cite this article as: Wang P, Gao B, Wang M, Sheng Q, Tu M. Challenges in the nursing care of intracranial carbapenem-resistant Escherichia coli infection after severe traumatic brain injury: a case report. Ann Palliat Med 2020;9(4):2381-2385. doi: 10.21037/apm20-1264 imipenem-colistin combination therapy against multipledrug-resistant Enterobacter cloacae: in vitro activity and a Galleria mellonella model. J Microbiol Immunol Infect 2018;51:70-5.

8. Zusman O, Altunin S, Koppel F, et al. Polymyxin monotherapy or in combination against carbapenemresistant bacteria: systematic review and meta-analysis. J Antimicrob Chemother 2017;72:29-39.

9. Davis K Jr, Johannigman JA, Campbell RS, et al. The acute effects of body position strategies and respiratory therapy in paralyzed patients with acute lung injury. Crit Care 2001;5:81-7.

10. Branson RD, Gomaa D, Rodriquez D Jr. Management of the artificial airway. Respir Care 2014;59:974-89.

11. Nunes LC, Rizzetti DA, Neves D, et al. Mechanical insufflation/exsufflation improves respiratory mechanics in critical care: Randomized crossover trial. Respir Physiol Neurobiol. 2019;266:115-20.

12. Kaiser JA, Westers JB. Nursing teamwork in a health system: A multisite study. J Nurs Manag 2018;26:555-62.

13. Silva YR, Greer TA, Morgan LC, et al. A Comparison of 2 Respiratory Devices for Sputum Clearance in Adults With Non-Cystic Fibrosis Bronchiectasis. Respir Care 2017;62:1291-7.

14. Yildirim E. Principles of Urgent Management of Acute Airway Obstruction. Thorac Surg Clin 2018;28:415-28.

15. Akova M, Daikos GL, Tzouvelekis L, et al. Interventional strategies and current clinical experience with carbapenemase-producing Gram-negative bacteria. Clin Microbiol Infect 2012;18:439-48.

16. Chen QH, Lin D, Yu QG, et al. Efficacy of lumbar cistern drainage combined with intrathecal antibiotherapy for the treatment of ventriculo-subarachnoid infections following surgery for hypertensive intracerebral hemorrhage. Neurochirurgie 2017;63:13-6.

(English Language Editor: J. Chapnick) 